\title{
Optimal dynamic performance for high-precision actuators/stages
}

\author{
Curt Preissner $^{* \mathbf{a}}$, Soon-Hong Lee ${ }^{\mathbf{a}}$, Thomas J. Royston ${ }^{\mathbf{b}}$, and Deming Shu ${ }^{\mathbf{a}}$ \\ ${ }^{\mathrm{a}}$ Advanced Photon Source, Argonne National Laboratory; ${ }^{\mathbf{b}}$ Dept. of Mechanical Engineering, \\ University of Illinois at Chicago
}

\begin{abstract}
System dynamic performance of actuator/stage groups, such as those found in optical instrument positioning systems and other high-precision applications, is dependent upon both individual component behavior and the system configuration. Experimental modal analysis techniques were implemented to determine the six degree of freedom stiffnesses and damping for individual actuator components. These experimental data were then used in a multibody dynamic computer model to investigate the effect of stage group configuration. Running the computer model through the possible stage configurations and observing the predicted vibratory response determined the optimal stage group configuration. Configuration optimization can be performed for any group of stages, provided there is stiffness and damping data available for the constituent pieces.
\end{abstract}

Keywords: vibration, modal analysis, high precision, multibody, optimization

\section{INTRODUCTION AND MOTIVATION}

The Advanced Photon Source (APS) at Argonne National Laboratory is a third-generation national synchrotron radiation light source research facility. Users of this facility carry out basic and applied research in the physical sciences, biological sciences, planetary sciences and innovative x-ray instrumentation. The ability of investigators to carry out their work rests on the assumption that the $\mathrm{x}$-ray beam, the sample it impinges upon, and the $\mathrm{x}$-ray detector remain stable, with known relative positions and orientations. As it requires considerable effort and resources to produce the $\mathrm{x}$ ray beam, maximizing the light use is essential. To this end, instrumentation engineers are introducing automated positioning systems and robots to reduce the need for manual manipulation of optics, samples and detectors and increase the beam utilization. Although this approach enables researchers to generate more data, the introduction of multibody flexible manipulators can confound data acquisition due to the increased structural flexibility of the system. Engineers within the Experimental Facilities Division (XFD) have undertaken projects to improve the structural stability of existing systems and ensure the structural stability of systems currently under design.

There exists a history of work to ensure optimal dynamic performance of x-ray components at the APS. It can be divided into three basic areas: ground motion studies ${ }^{1,2}$, existing equipment analysis and remediation ${ }^{3-9}$, and new design consideration $^{6,7}$. The current work investigates a recently implemented robotic x-ray sample handling system with the intent of developing modeling techniques to aid future designs. The objective of this research is to further the application of multibody vibration and multibody dynamics in improving the performance of support and positioning structures at the APS. Specific goals include identifying component stiffness and damping properties, construction of finite element (FE) multibody vibration models of the positioning systems, comparison of the model to experimentally measured behavior, modification of the FE model to include the change in stage configuration and eventual implementation of a large displacement multibody dynamic model of the robot system.

The subject of this research is a 3-axis Cartesian robotic manipulator, which is to be installed in a hutch on the 2-BM beamline of the Synchrotron Radiation Instrumentation Collaborative Access Team (SRI-CAT) to aid high throughput research. Figure 1 illustrates the layout of the robot system. The robot consists of three orthogonally oriented linear stages, with an end effector attached to the end of the Y stage. This particular robot will fulfill a pick-and-place type mission, moving samples from a magazine to the target area in the beamline. For this specific application, the dynamic performance during sample transit is not an issue, so the manipulator structure was not designed with dynamic stability in mind. Even though the dynamic stability of the robot's path is not critical for this application, we changed the

* preissne@aps.anl.gov; phone 1630252 3020; fax 1630252 9350; http://www.aps.anl.gov;

Argonne National Laboratory 9700 S. Cass Avenue, Argonne, IL 60439-4800 
connection between the $\mathrm{X}$ and $\mathrm{Z}$ stages. The original design called for an $\mathrm{X}$ stage with dual carriages; however, due to part unavailability, the system was constructed with a single carriage $X$ stage. The difference between the two stages is shown in Figure 2. Subsequently the dual carriage X stage became available, and, before system commissioning, the single carriage actuator will be replaced. The focus of this paper is modeling the device, both before and after changing the configuration of the $\mathrm{X}$ stage. This robot was chosen for the study due to its similarity to proposed systems, and the availability of a duplicate device in an off-beamline laboratory; thus it should be an excellent subject for application of multibody system theory.

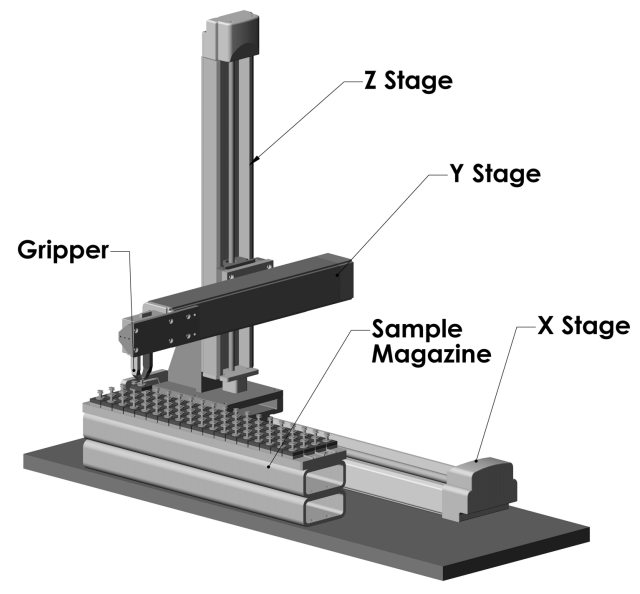

Figure 1: Cartesian robotic manipulator.
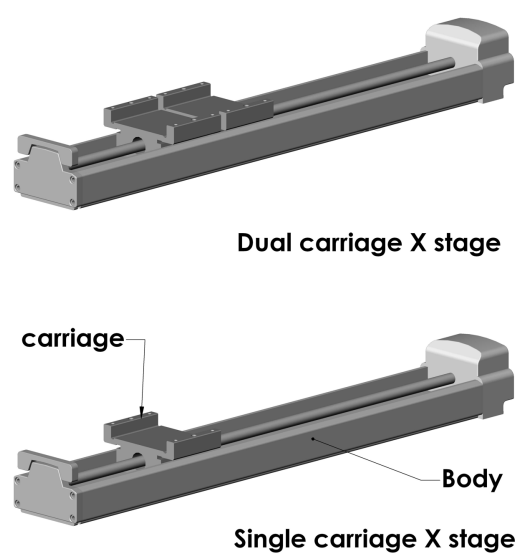

Figure 2: Comparison of $\mathrm{X}$ stages.

\section{METHODOLOGY}

The procedure used to construct and validate a multibody model and modify the model to account for the replacement of the $\mathrm{X}$ stage of the Cartesian positioner is described in the following. First, individual components of the XYZ manipulator were examined using experimental modal analysis to determine the modal parameters of mass, stiffness and damping. Next, a FE model was constructed using ANSYS 5.7, and the before mentioned modal parameters were input into the model. An experimental modal analysis of the complete robot system was then performed for confirmation of the FE model behavior. Finally, the FE model was modified to take into account the two-carriage X stage.

An experimental modal analysis is the process by which the natural frequency, stiffness and damping properties of a system can be determined from experimentally obtained frequency response functions (FRF). Classical vibration theory allows a multi degree of freedom system (MDOF) to be considered as an equivalent system of uncoupled, second order differential equations, each representing a single degree of freedom system. ${ }^{10}$

The SDOF system governing differential equation is:

$$
m \ddot{x}+c \dot{x}+k x=f .
$$

The excitation and response are assumed to be harmonic, with frequency $\omega$ :

$$
f=F e^{i \omega t} \quad x=X e^{i \omega t} .
$$

The FRF can be found by substituting equations (2) into equation (1), canceling the time-varying component, and rearranging:

$$
\begin{gathered}
H(\omega)=\frac{X(\omega)}{F(\omega)}=\frac{1 / m}{-\omega^{2}+i c \omega \frac{1 / m}{}+k 1 / m}=\frac{1 / m}{(i \omega-\lambda)\left(i \omega-\lambda^{*}\right)}, \\
\lambda=\sigma+i \omega_{d} \quad \lambda^{*}=\sigma-i \omega_{d}
\end{gathered}
$$


where $X(\omega)$ is the displacement output and $F(\omega)$ is the force input. Equations (4) are the complex conjugate roots containing the damping coefficient, $\sigma$, and the damped natural frequency, $\omega_{d}$. For modal parameter estimation, it is convenient to expand equation (3) in terms of partial fractions:

$$
H(\omega)=\frac{A}{(i \omega-\lambda)}+\frac{A^{*}}{\left(i \omega-\lambda^{*}\right)} .
$$

Constants $A$ and $A^{*}$ are the called the FRF residues; they are related to the mode shape and modal mass. Multi degree of freedom and continuous systems can be viewed as a linear superposition of these SDOF frequency response functions. ${ }^{11}$

In order to determine the modal parameters from experimental data, modal analysis software, such as Vibrant Technology, Inc.,'s ME-Scope is employed. The software is used to fit a polynomial to the denominator of the experimentally determined FRF; frequency and damping are estimated by finding the roots to this polynomial.

The FRFs were generated through impact testing. Accelerometers were mounted at a fixed location to the stage or structure under test. Impacting the structure at a number of points with a force transducer equipped hammer provided the excitation. The FRF between the force excitation input and the acceleration response output were measured using Data Physics 620 Analyzer software running on an HP E1432 VXI analyzer. Once FRFs for all of the desired measurement locations were collected, the data were then transferred to ME Scope for analysis. In ME Scope, the FRFs were summed together to yield one representative function used to determine the frequency bands in which to curve fit. Natural frequency, modal damping, and mode shape were all determined from the curve fit data. The behavior of the structure for a particular mode shape was visualized using the animation capability of ME Scope. The visual assistance of animation was necessary for two reasons: to identify mode shapes for stiffness calculation and to compare the FE model with the experimental results.

Stiffness estimation between the stage body and the stage carriage was an important step in building the FE model. Once the impact modal data were processed with ME Scope, specific mode shapes could be considered. The intent was to find three modes where translational motion between the carriage and the stage body dominated the response (see Figure 3 ). Damping and stiffness values would be selected from these mode shapes.

\section{Y Direction}

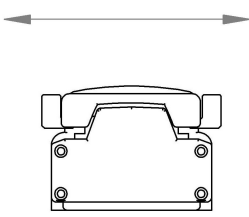

\section{Direction}
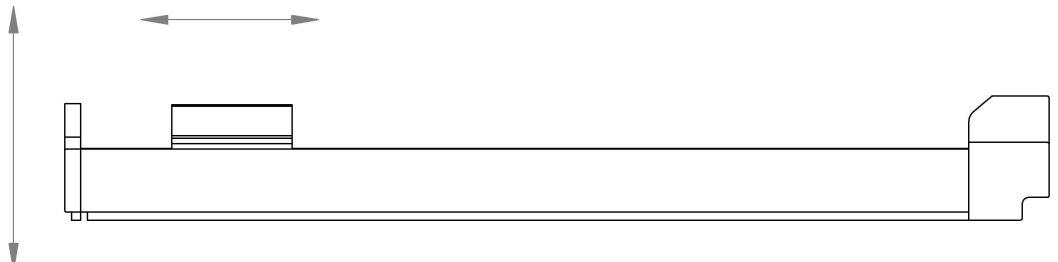

Z Direction

Figure 3: Desired vibration directions.

From these modes, stage stiffness in the X, Y and Z directions could be estimated using SDOF theory. Stiffness, $k$, can be found in the following manner: 
Natural frequency is defined as:

$$
\omega=\sqrt{k / m} .
$$

Stiffness can be determined to be:

$$
k=m(2 \pi f)^{2},
$$

where $m$ is the mass of the stage in kilograms, and $f$ is the mode of interest natural frequency in hertz. It was not possible to resolve three translational modes for each of the stages tested, though enough information was provided to build an accurate FE model. For the X stage carriage, two translational modes were identified. For the Y stage, one translational mode was used to determine the stiffness.

With the necessary stiffness data in hand, the construction of a model for FE analysis could proceed in ANSYS 5.7. Three bodies comprised the simplified FE model: the bracket between the $\mathrm{X}$ stage and $\mathrm{Z}$ stage, the $\mathrm{Z}$ stage, and the $\mathrm{Y}$ stage. Keeping in mind that the behavior of stage interconnection was of primary concern, the bracket was modeled and meshed using its exact geometry and material properties. The two stages were considered rigid and replaced with rigid bodies possessing similar mass and geometric properties. The $\mathrm{X}$ stage was not included in the model, as it was considered rigid and constrained by the ground. The model geometry is illustrated in Figure 4.

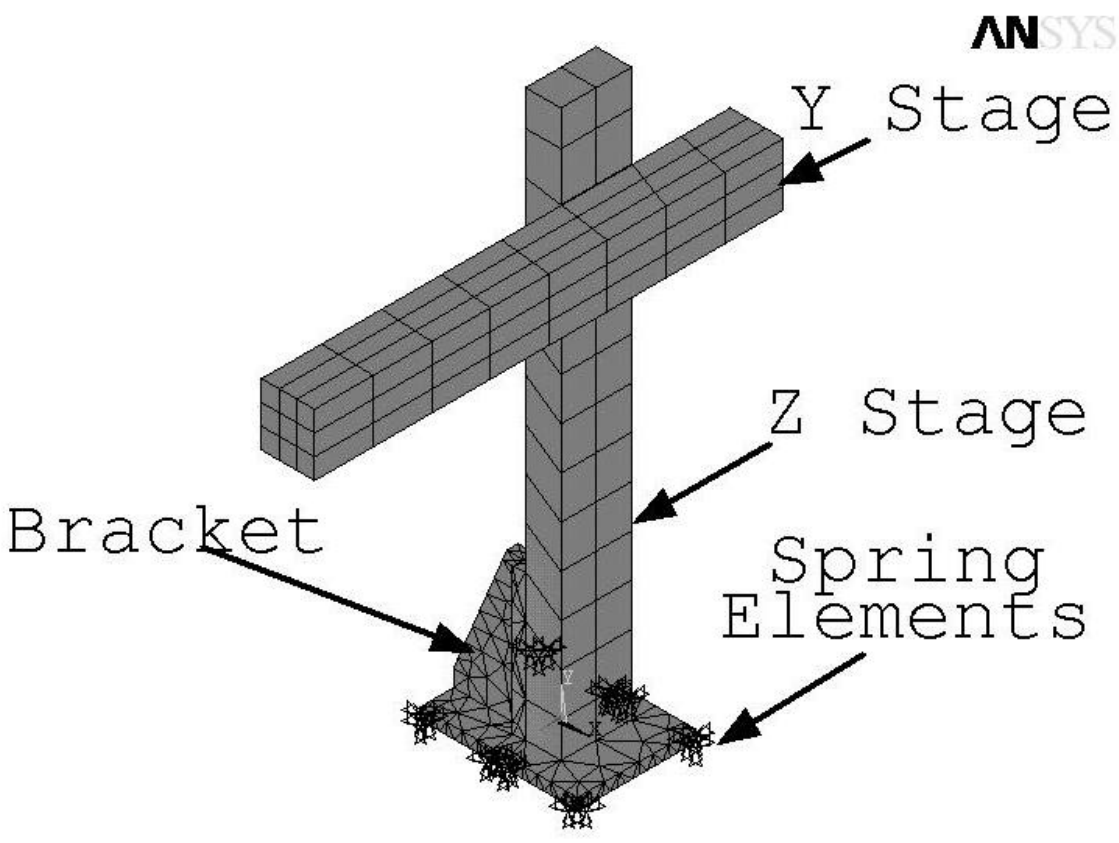

Figure 4: FE model geometry.

The connection between the bracket and the $\mathrm{Z}$ stage was rigid, that is both bodies shared common node points. Experimentally determined stiffnesses were applied between the bracket and ground, and the $\mathrm{Z}$ stage and $\mathrm{Y}$ stage. For the bracket-ground connection, the experimentally determined damping and stiffness were applied using massless spring/damper elements (COMBIN14) that act in only one specified degree of freedom (DOF). In order to represent the single-carriage $\mathrm{X}$ stage, the three stiffnesses and the three damping coefficients as determined from the two translational modes were applied to the base of the bracket. One quarter of each stiffness and damping value was applied at one of four points, with the total values equaling that of the modal analysis determined stiffness and damping. Figure 5 describes the orientation and position of the single-carriage spring/damper elements. The two-carriage connection was similar to the single-carriage model, with the addition of another group of spring/damper elements to the base of the bracket. Figure 6 shows a close up of three stiffness elements. 

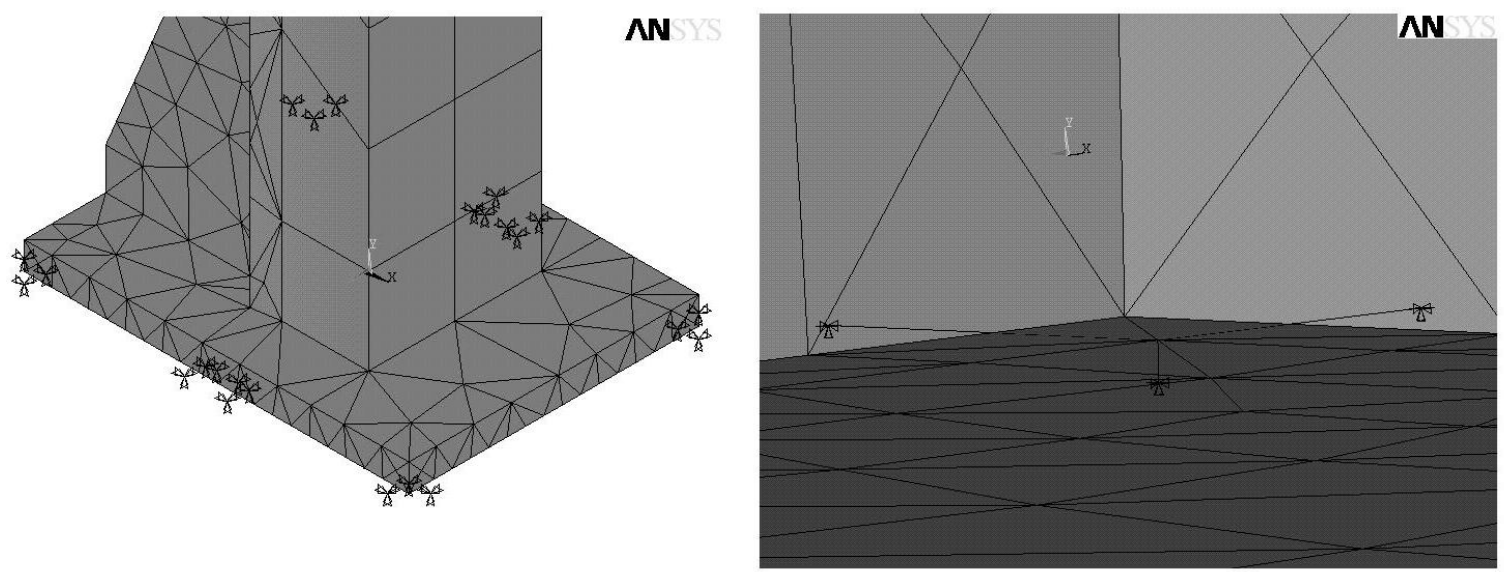

Figure 5: Single-carriage and double-carriage stiffness application.

Figure 6: Close-up of carriage stiffness configuration.

From the Y stage modal analysis results, we decided to assume equal stiffness in the three translational directions. In the FE model these stiffnesses were represented with a single massless spring/damper element (COMBIN14) with a three DOF behavior. The assumption of small displacements allowed a system of three orthogonally arranged springs to be approximated with a single spring element

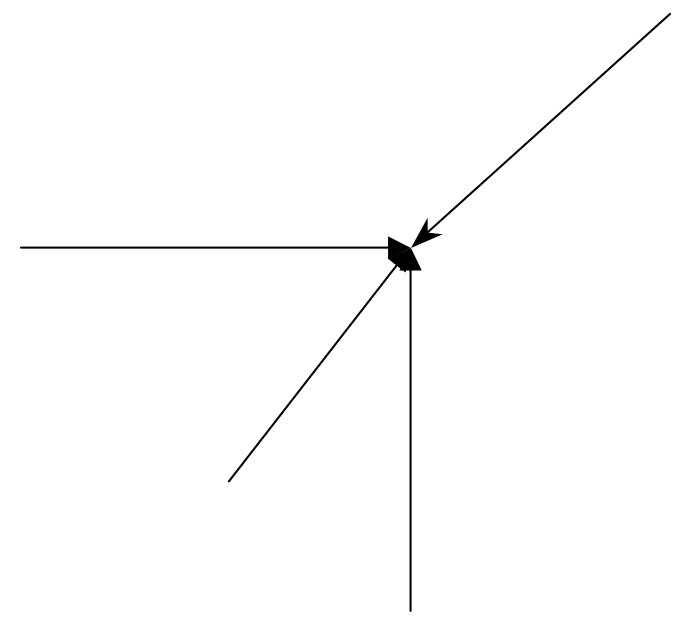

Figure 7: Equivalent force system.

Figure 7 illustrates two sets of equivalent forces: orthogonally arranged forces, $\mathbf{f}$, are balanced by the equivalent force, $\mathbf{F}$. If each of the $\mathbf{f}$ forces are due to a spring, $\mathbf{k}$, then $\mathbf{F}$ can be due to an equivalent spring, $\mathbf{K}$. The following can then be assumed.

$$
F=\sqrt{(f)^{2}+(f)^{2}+(f)^{2}}=\sqrt{3(f)^{2}},
$$

also

$$
f=k x \text { and } F=K \Delta X \text {, }
$$

so

$$
K \Delta X=\sqrt{3(k x)^{2}}
$$




$$
\begin{aligned}
& \frac{K \Delta X}{\sqrt{3}}=k x, \text { where } x \approx \Delta X \\
& \therefore \quad K \approx k \sqrt{3} .
\end{aligned}
$$

The experimental modal analysis of the complete manipulator system was performed in a similar fashion as that of the $\mathrm{X}$ and Y stages. The data was curve fit using ME Scope, which was then used to visualize the mode shapes for comparison of the FE model. FE model modal analysis was carried out using ANSYS. Conformation of the model stiffness parameters was obtained by comparing the FE natural frequencies and mode shapes to the experimental natural frequencies and mode shapes. When agreement was achieved, the final step was taken; the FE model was modified to the dual-carriage configuration.

\section{DATA AND RESULTS}

\subsection{X stage modal analysis and stiffness identification}
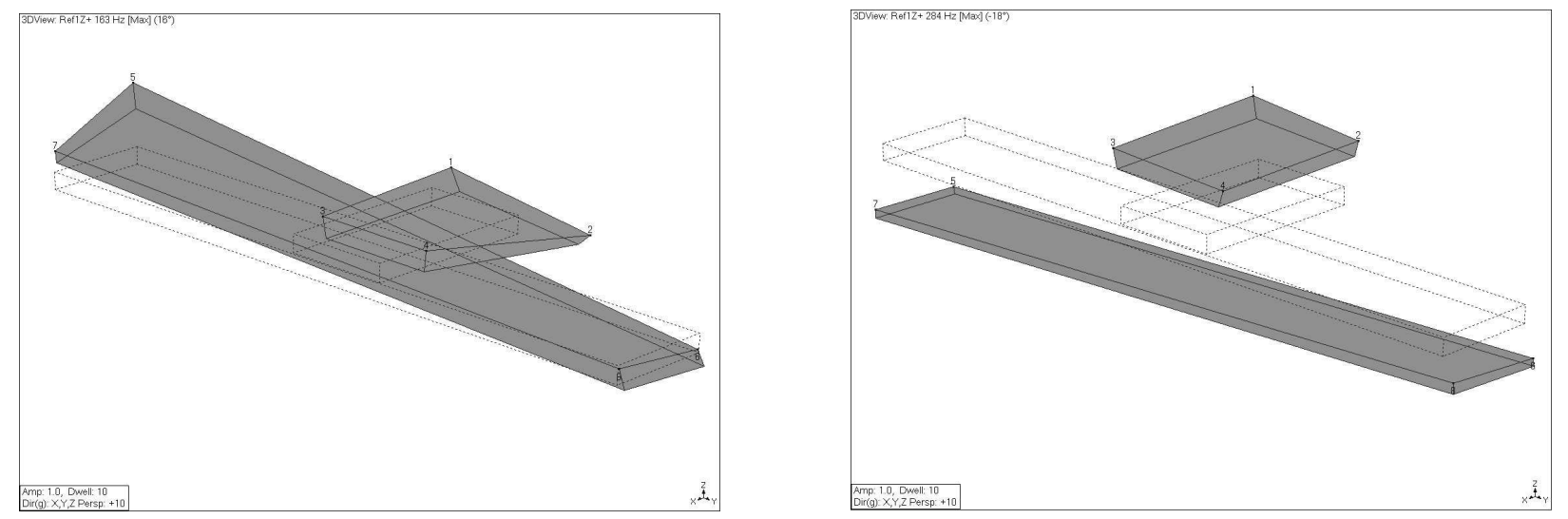

Figure 8: $163 \mathrm{~Hz}$ (left) and $284 \mathrm{~Hz}$ (right) experimental modes used to determine $\mathrm{X}$ stage stiffness.

The two modes shown in Figure 8 were used to determine the $X$ stage stiffness. The mode on the left $(163 \mathrm{~Hz})$ was used to calculate the two in-plane stiffnesses, and the mode on the right $(284 \mathrm{~Hz})$ was used to calculate the transverse stiffness. Table I contains the calculated stiffness and damping for the $\mathrm{X}$ stage.

Table I: X stage stiffness.

\begin{tabular}{|c|c|c|}
\hline Modal Frequency $(\mathrm{Hz})$ & Calculated Stiffness $(\mathrm{N} / \mathrm{m})$ & Calculated Damping $(\mathrm{N} \bullet \mathrm{s} / \mathrm{m})$ \\
\hline 163 & $11.5 \mathrm{E} 6$ & 1705 \\
\hline 284 & $29.6 \mathrm{E} 6$ & 530 \\
\hline
\end{tabular}




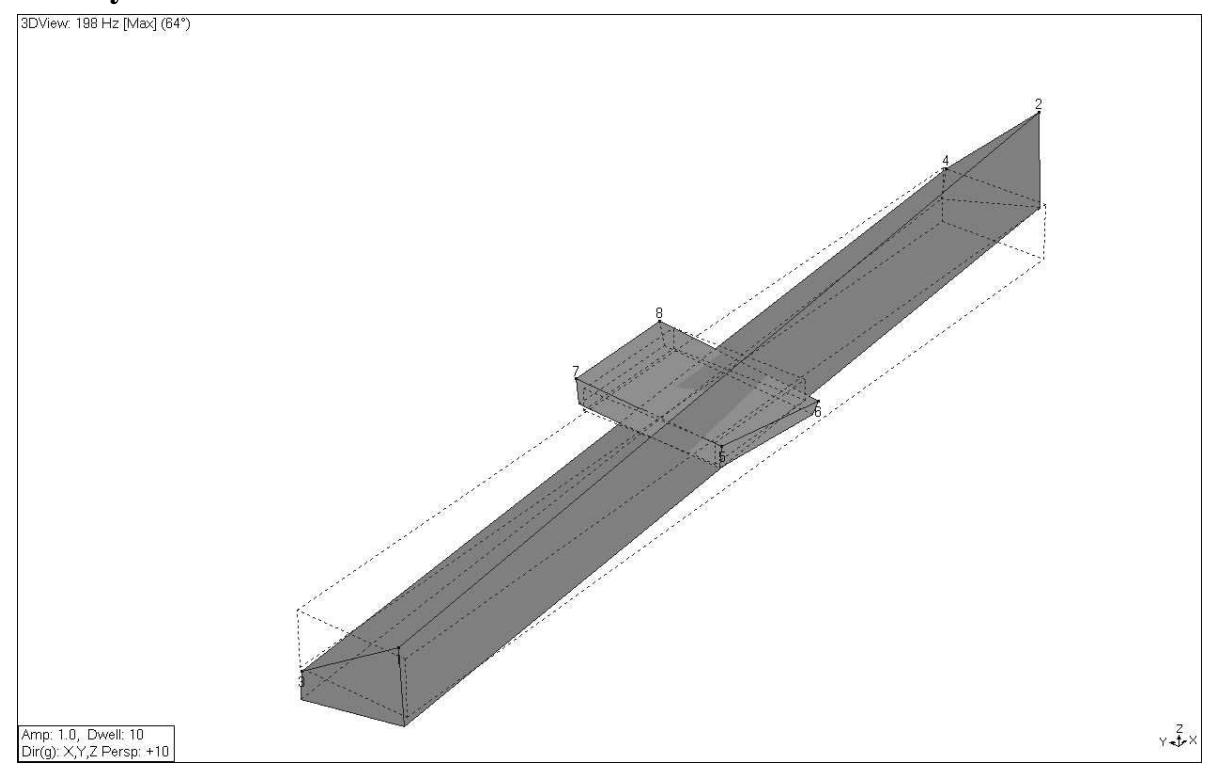

Figure 9: $198 \mathrm{~Hz}$ Experimental mode used to determine Z stage stiffness.

A single experimentally determined mode was used to calculate the $Z$ stage stiffness (see Figure 9). The same stiffness was assumed for all three directions. Table II contains the calculated stiffness and damping for the $\mathrm{Z}$ stage.

Table II: Y stage stiffness.

\begin{tabular}{|c|c|c|}
\hline Modal Frequency $(\mathrm{Hz})$ & Calculated Stiffness $(\mathrm{N} / \mathrm{m})$ & Calculated Damping $(\mathrm{N} \bullet \mathrm{s} / \mathrm{m})$ \\
\hline 198 & $7.2 \mathrm{E} 6$ & 1187 \\
\hline
\end{tabular}

\subsection{Single-carriage FE model}

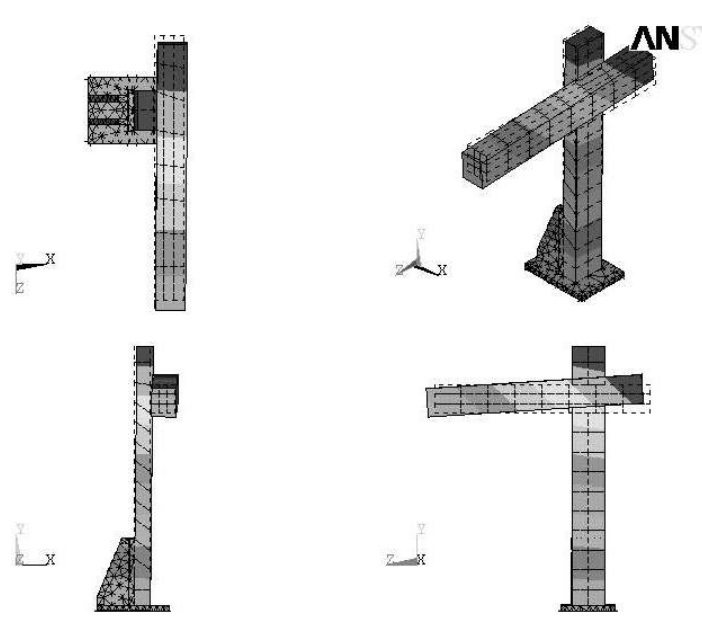

Figure 10: Single-carriage configuration, $25.3 \mathrm{~Hz}$ mode.
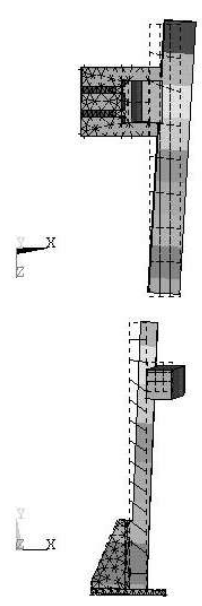
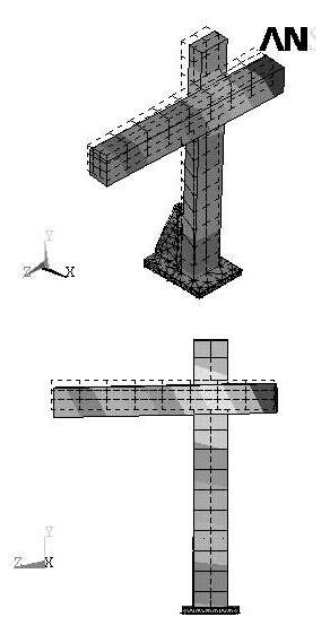

Figure 11: Single-carriage configuration, $43.5 \mathrm{~Hz}$ mode.

Inspection of the FE $25.3 \mathrm{~Hz}$ mode (Figure 10) indicates a rocking of the Y stage in the Z-Y plane (FE coordinate system), and a rocking of the $\mathrm{Z}$ stage and bracket in the $\mathrm{X}-\mathrm{Y}$ plane. The $43.5 \mathrm{~Hz}$ mode (Figure 11) displays rotation of the $\mathrm{Y}$ stage about the $\mathrm{Z}$ stage in the $\mathrm{X}-\mathrm{Z}$ plane and rocking of the $\mathrm{Z}$ stage and bracket in the $\mathrm{X}-\mathrm{Y}$ plane. 
3.4 Robot experimental modal analysis

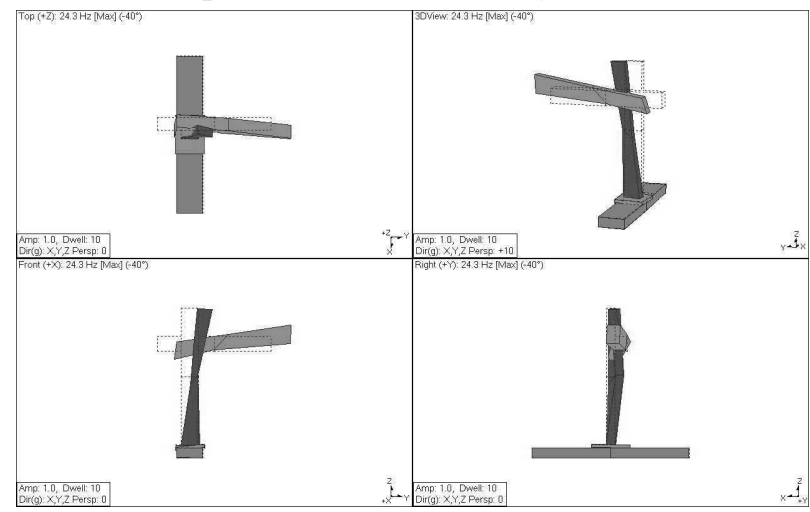

Figure 12: Single carriage configuration, $24 \mathrm{~Hz}$ mode.

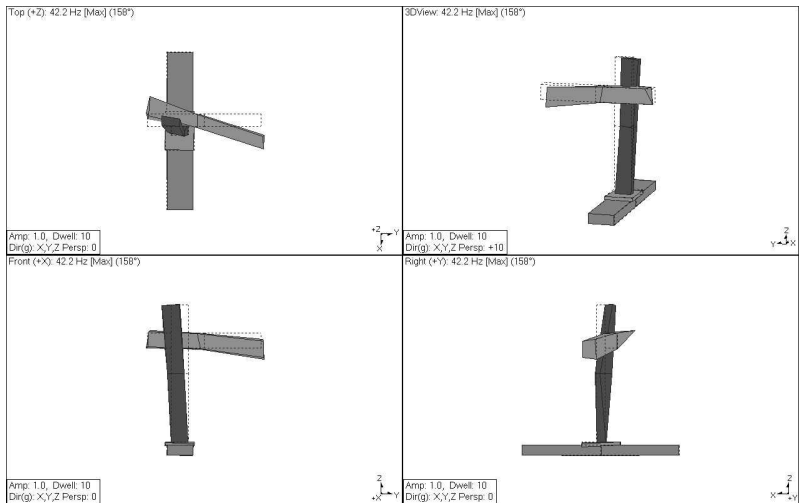

Figure 13: Single carriage configuration, $42 \mathrm{~Hz}$ mode.

Note that each of the modes shown in Figures 12 and 13exhibits a similarity to the FE results. This is evident by comparing the motion of the $\mathrm{Y}$ stage and the $\mathrm{Z}$ stage bodies.

\subsection{Dual-carriage FE model}
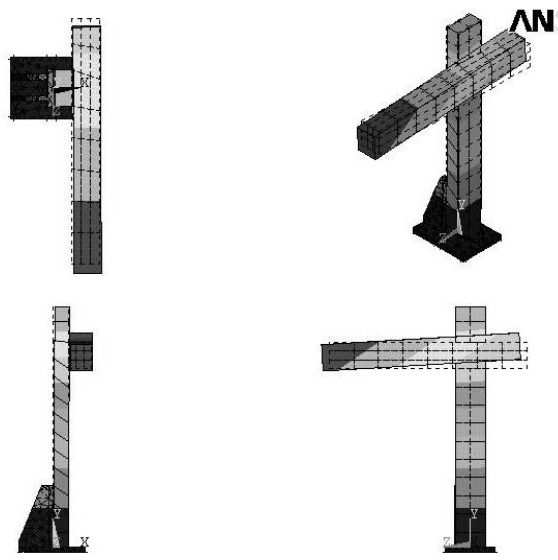

Figure 14: $26.9 \mathrm{~Hz}$ mode.
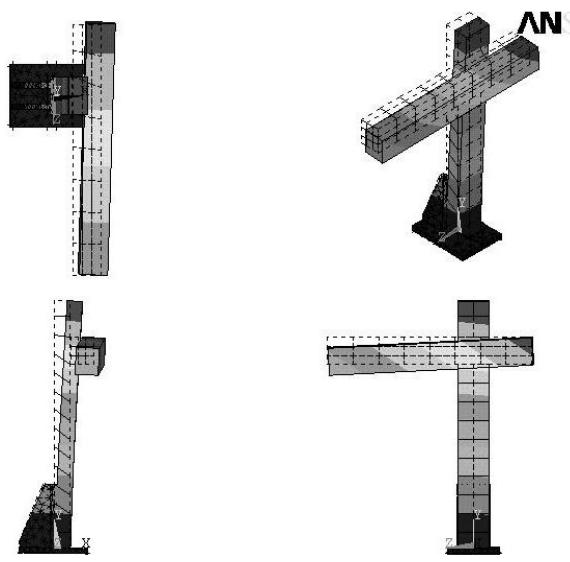

Figure 15: $57.6 \mathrm{~Hz}$ mode.

Figure 14 and Figure 15 are the mode shapes predicted by the FE model for the dual-carriage configuration. They are similar in motion to Figures 11 and 12, although shifted up the frequency spectrum.

\section{CONCLUSIONS}

As stated above, for the single-carriage stage, the experimental modal analysis results agree with the FE modal analysis results. This agreement indicates that the experimental determination of the component stiffnesses was reasonable. Extending this idea, one can conclude that the behavior predicted by the FE model for the dual carriage stage is reasonable as well. The benefit of the shift in frequency of the two modes should be a decrease in amplitude of the motion at the gripper. A harmonic analysis can be performed to determine the gripper motion as a function of base excitation amplitude.

With respect to additional work, the identification of component properties as discussed in this paper is necessary to move on to more complex models. Although the vibrational model, which forms the basis of this paper, is sufficient for static or quasi-static applications, it is insufficient for modeling the behavior of the robot while in motion. A multibody dynamic model is necessary to account for the large displacements and configuration changes encountered if Cartesian or revolute joint robots are to be modeled while in motion. Key to the application of multibody software is the insertion of the component parameters: inertia, stiffness, and damping. Additional consideration may need to be given in the form 
of flexible bodies, as the stages have the capability of producing large accelerations. These further steps may be considered to realize the robotic manipulation of $\mathrm{x}$-ray detectors and samples.

\title{
ACKNOWLEDGEMENTS
}

Advanced Photon Source, Argonne National Laboratory

D. Shu

S. Lee

\section{University of Illinois at Chicago, Chicago, IL}

\author{
T. J. Royston
}

This work was supported by the U. S. Department of Energy, Office of Science under Contract No. W-31-109-Eng-38.

\section{REFERENCES}

1. Jendrzejczyk, J. A., M. W. Wambsganss, R. K. Smith. (1990).”Surveillance of $18 \mathrm{~Hz}$ components of ground motion at the APS site." Argonne National Laboratory, unpublished.

2. Jendrzejczyk, J. A., M. W. Wambsganss, R. K. Smith. (1992). "Surface wave attenuation characteristics at the APS site.” Argonne National Laboratory, unpublished.

3. Jendrzejczyk, J. A., M. W. Wambsganss, R. K. Smith. (1992). "Vibration study of the APS storage ring 0.8 meter quadrupole maget/magnet support assembly." Argonne National Laboratory, unpublished.

4. Chen, S. S., S. Zhu, M. W. Wambsganss, J. A. Jendrzejczyk. (1994). "Identification of root cause of vibration of a liquid gallium-cooled silicon monochromator and recommendations for abatement. Argonne National Laboratory, unpublished.

5. Basdogan, I. (1997). "A theoretical and experimental study of the dynamic response of high precision optical positioning systems." Ph.D. diss., Department of Mechanical Engineering, University of Illinois at Chicago.

6. Basdogan, I, T. J. Royston, A. A. Shabana, D. Shu, T. Kuzay. (1996). "Vibratory response of a mirror support/positioning system for the Advanced Photon Source Project at Argonne National Laboratory." Proc. SPIE 2865, 2-13.

7. Barraza, J., D. Shu, T. Kuzay, T. J. Royston. (1999). "Vibratory response modeling and verification of a highprecision optical positioning system." Proc. SPIE 3786, 86-97.

8. Barraza, J., D. Shu, T. Kuzay, T. J. Royston. (1998). "Vibratory response of a precision double-multilayermonochromator positioning system using a generic modeling program with experimental verification." Proc. SPIE 3429, 265-270.

9. Lee, S. H., C. Preissner, B. Lai, Z. Cai, D. Shu. (2002) "Enhancement of the vibration stability of a microdiffraction goniometer." This volume.

10. Shabana, A. A. (1991). Theory of Vibration, Volume II: Discrete and Continuous Systems, Chapter 3. New York: Springer - Verlag, Inc.

11. Allemang, R., Brown, D. (1996). "Experimental Modal Analysis,” In C. Harris (Ed.), Shock and Vibration Handbook (pp. 21.1-21.74). New York: McGraw Hill. 